Meta

Journal des tradlucteurs

Translators' Journal

\title{
Notions fondamentales de mécanique
}

\section{Gilles Bélanger}

Volume 34, numéro 4, décembre 1989

URI : https://id.erudit.org/iderudit/002031ar

DOI : https://doi.org/10.7202/002031ar

Aller au sommaire du numéro

Éditeur(s)

Les Presses de l'Université de Montréal

ISSN

0026-0452 (imprimé)

1492-1421 (numérique)

Découvrir la revue

Citer cet article

Bélanger, G. (1989). Notions fondamentales de mécanique. Meta, 34(4), 752-764.

https://doi.org/10.7202/002031ar d'utilisation que vous pouvez consulter en ligne.

https://apropos.erudit.org/fr/usagers/politique-dutilisation/ 


\section{NOTIONS FONDAMENTALES DE MÉCANIQUE}

\section{INTRODUCTION}

Quand on parle de mécanique, la plupart des gens pensent aussitôt à la mécanique automobile ou à quelque chose qui s'en approche. C'est bien normal, quand on songe que la voiture est peut-être le seul objet qui fasse l'envie d'à peu près tout le monde depuis une quarantaine d'années. Il n'empêche qu'aborder cette discipline sous ce seul aspect, c'est un peu masquer un certain nombre de notions fondamentales qui peuvent apporter un éclairage intéressant tant à la mécanique automobile qu'à d'autres branches de la mécanique appliquée, par exemple les constructions mécaniques ou la robotique.

Bon nombre de notions fondamentales de mécanique ont un contenu assez flou dans l'esprit des non-spécialistes. Les machines sont, par exemple, des «appareils», des «dispositifs» remplaçant l'homme dans l'exécution des tâches les plus diverses. Ce sont, sans trop exagérer, des «boîtes mystérieuses» d'où sortent des dispositifs faisant tourner une poulie ou pouvant recevoir des outils de toutes sortes.

Nous n'aborderons ici que certaines notions qu'il faut connaître pour comprendre les descriptions de machines bien réelles et leur fonctionnement. Les notions, plus fondamentales encore, de la mécanique théorique : cinématique (kinematics), cinétique (kinetics), statique (statics), dynamique (dynamics), etc.; elles seront abordées dans un prochain article.

\section{NOTIONS FONDAMENTALES}

Au moment d'aborder l'étude des machines, il n'est pas superflu d'essayer de les définir autrement que par l'aspect immédiatement utilitaire que nous leur attribuons tous de prime abord. Une définition que l'on peut imaginer serait la suivante: «Les machines sont des assemblages de pièces combinées de façon à recevoir une forme donnée d'énergie, à la transformer et à la restituer sous une forme appropriée.» Voilà une définition qui s'applique aussi bien aux machines, au sens classique du terme, qu'aux «machines électroniques», qui ne comportent pas de pièces mobiles ou qui, en tout cas, n'ont pas pour fonction principale de transformer une énergie en une autre, mais plutôt de traiter des signaux. Un micro-ordinateur, par exemple, répond parfaitement à la définition donnée plus haut. Il ne viendrait toutefois à personne l'idée de les classer parmi les constructions mécaniques. Les machines qui intéressent la mécanique ont par contre la particularité de mettre en jeu des forces (force) et des mouvements (motion). Ces caractéristiques nous serviront à les distinguer des autres types de «machines».

Cela nous amène donc à proposer une nouvelle définition, plus restrictive : «Les machines sont des systèmes mécaniques qui reçoivent de l'énergie d'une source quelconque, la transforment et la transmettent sous une autre forme (énergie ou mouvernent) à un organe récepteur en vue d'effectuer un certain travail mécanique.» Ces considérations nous amènent à préciser d'autres notions, plus fondamentales encore.

\subsection{MOUVEMENT}

L'idée de mouvement (motion) suppose le déplacement d'un corps d'une position relative $\mathbf{a}$ à une position relative $\mathbf{b}$ entre un instant $\mathbf{x}$ et un instant $\mathbf{y}$. On $\mathbf{y}$ associe donc une distance (distance) et une durée (time). Sous-jacentes à ces notions se trouvent celles de trajectoire (trajectory), de vitesse (speed) et d'accélération (acceleration). Nous n'examinerons pas ici ces notions, et d'autres encore, qui relèvent de l'étude théorique des corps au repos et en mouvement, c'est-à-dire des disciplines dont nous avons parlé plus haut. 
Pour les besoins de cet article, disons simplement qu'il existe différents types de mouvements, suivant le point de vue envisagé. Un mouvement peut être rectiligne (straight), si sa trajectoire est une droite, circulaire (circular, rotary) s'il décrit des cercles ou des arcs de cercle, sinusoïdal (sinusoidal), cycloidal (cycloidal), etc.; un mouvement est continu (continuous) s'il ne change pas de sens pendant l'intervalle de temps considéré, discontinu (discontinuous) dans le cas contraire; s'il est discontinu, il peut être alternatif (alternating, reciprocating) ou aléatoire (random); il peut encore être uniforme (uniform) si la distance parcourue est la même pour un même intervalle de temps; ou varié (variable) si la distance parcourue varie avec le temps: il sera accéléré (accelerated) ou bien décéléré, ou retardé (decelerated), suivant que la vitesse du mobile augmente ou diminue. Ainsi, dans un moteur automobile, les pistons décrivent un mouvement rectiligne alternatif ([straight] reciprocating motion) et l'arbre moteur, un mouvement circulaire continu (circular continuous motion); ce mouvement est uniforme si le moteur tourne à régime constant. Un mouvement possède une direction, qui est celle de l'orientation du déplacement, un sens, qui est celui dans lequel s'exerce la cause du mouvement, et une intensité, assimilable à la vitesse du déplacement.

\subsection{FORCE, TRAVAIL, PUISSANCE}

Il existe en mécanique un certain nombre de notions premières, ou fondatrices, qui tirent leur origine de la philosophie et dont découlent d'autres notions. La notion d'énergie en est une, tout comme celle de force.

Le Dictionnaire encyclopédique Larousse L1 (1979), par exemple, définit l'énergie (energy) comme la «faculté que possède un système de corps de fournir du travail mécanique ou son équivalent». Le Petit Robert, quant à lui, y voit «ce que possède un système s'il est capable de produire du travail». Le Dictionnaire encyclopédique Quillet (1968) propose par ailleurs ce qui suit: «L'énergie, au sens que lui donne la physique, est une notion abstraite, liée à toutes les manifestations de force, de mouvement, de chaleur, de champ de gravitation, de champ électrique ou magnétique, etc. La notion d'énergie, considérée d'abord en mécanique comme l'aptitude d'un corps à fournir, dans un système donné, une quantité de travail déterminée...» Pour tous ces dictionnaires, l'énergie est donc une notion abstraite, une faculté, une aptitude. Pour pousser plus loin, le physicien doit ici faire appel à la philosophie.

Il en est de même pour la notion de force (force, effort). Le Petit Robert nous dit que ce terme désigne «toute cause capable de déformer un corps, ou d'en modifier le mouvement, la direction, la vitesse». Nombre d'ouvrages didactiques la définissent encore comme la «cause qui tend à mouvoir un corps». D'ailleurs, le Dictionnaire encyclopédique Quillet n'aborde-t-il pas la définition de ce terme que dans la partie philosophique de son article, pour affirmer que «Le sens intuitif, et très vivace, de cette notion est celui de cause de mouvement.» Ici encore, nous nous trouvons devant une autre notion philosophique, celle de «cause». Il va sans dire que nous n'irons pas plus loin sur cette voie et que nous renvoyons le lecteur curieux à d'autres sources que celles que nous envisageons pour notre propos. Disons simplement que l'idée de force découle de celle d'effort musculaire: tout ce qui tend à produire le même effet que notre effort sur les corps peut être considéré comme une force.

Préalable à la notion de force se trouve toutefois celle d'action (action). Un corps peut exercer une action mécanique sur un autre corps, qui subit alors une déformation, un changement de direction ou une variation du mouvement (freinage, accélération ou immobilisation). Si les deux corps sont en contact, par exemple les gaz de combustion avec le piston ou la roue avec le sol, on parle d'action de contact (action by contact, contact action). Une action à distance (action at a distance) peut aussi s'exercer sur 
deux corps qui ne sont pas en contact; le magnétisme et l'attraction terrestre en sont deux exemples.

À la notion d'action s'oppose celle de réaction (reaction). Si un corps a exerce une force (action) sur un corps $\mathbf{b}$, ce dernier exerce en retour une action directement opposée (réaction) sur le premier. Tout comme le mouvement, les forces possèdent trois caractéristiques essentielles, qui définissent leur vecteur (vector): une direction, un sens et une intensité.

Une troisième notion essentielle est celle de travail (work). Déjà, nous abordons des rivages plus certains. Le travail, nous dit le Petit Robert dans une dernière définition, c'est le «produit d'une force par le déplacement de son point d'application (estimé suivant la direction de la force)». C'est la même définition que fait valoir le Dictionnaire encyclopédique Quillet, qui précise: «À l'origine du concept scientifique de travail se trouve la notion usuelle de travail, elle-même liée directement à la notion de fatigue. Nous dirons d'une force qu'elle travaille si son point d'application se déplace; ainsi, un homme qui supporte une charge et la tient immobile se fatigue, mais ne produit aucun travail (au sens physique du terme); c'est qu'à l'idée intuitive de travail correspondent en fait deux concepts scientifiques distincts: le travail (produit d'une force par un déplacement) et l'action (produit d'une force par un temps).» En effet, une force qui déplace une masse fournit un travail positif, ou travail moteur (motive work), tandis que la masse y oppose un travail négatif, ou travail résistant (resistive work). Une force appliquée à un corps mais ne le déplaçant pas n'effectue aucun travail: elle exerce simplement une action sur ce corps.

Un système mécanique qui fournit un travail possède de l'énergie: énergie cinétique (kinetic energy) si cette énergie est due à un mouvement; énergie potentielle (potential energy) si elle résulte de la position du système par rapport à la terre (la pomme de Newton), etc. Les notions de travail et d'énergie sont donc très proches.

Une autre notion fondamentale en mécanique est celle de puissance (power, output, work output), que l'on confond dans la langue courante tantôt avec la notion de force, tantôt avec celle de travail. La puissance est tout simplement la quantité de travail fournie par unité de temps. Enfin, le rendement (efficiency, power efficiency) n'est, quant à lui, que le rapport de la puissance utile (useful power output) à la puissance absorbée (power input, input). Le rendement s'exprime le plus souvent par un pourcentage ou par un nombre adimensionnel inférieur à l'unité : la puissance utile fournie par la machine est en effet inférieure à la puissance absorbée, c'est-à-dire celle qui est fournie à la machine, à cause des pertes dues aux frottements (friction loss), à l'échauffement, etc.

Le tableau suivant met en relation les différentes grandeurs vues jusqu'ici. Les unités mentionnées sont celles du système international (SI). 
Tableau 1

Grandeurs mécaniques fondamentales

\begin{tabular}{|c|c|c|c|c|}
\hline Grandeur & Équation & Dimensions & Unité & $\begin{array}{l}\text { Unités } \\
\text { constitutives }\end{array}$ \\
\hline Force & $\mathrm{F}=\mathrm{ma}$ & $\mathrm{ML} \mathrm{T}^{-2}$ & newton & $1 \mathrm{~N}=1 \mathrm{~kg} \cdot \mathrm{m} / \mathrm{s}^{2}$ \\
\hline Travail & $W=F d$ & $\mathbf{M L}^{2} \mathrm{~T}^{-2}$ & joule & $1 \mathrm{~J}=1 \mathrm{~N} . \mathrm{m}$ \\
\hline $\begin{array}{l}\text { Énergie } \\
\text { cinétique }\end{array}$ & $E=1 / 2 \mathrm{mv}^{2}$ & $M \mathbf{L}^{2} \mathrm{~T}^{-2}$ & joule & $1 \mathrm{~J}=1 \mathrm{~kg}(\mathrm{~m} / \mathrm{s})^{2}$ \\
\hline $\begin{array}{l}\text { Énergie } \\
\text { potentielle }\end{array}$ & $E=m g h$ & $M L^{-2} \mathrm{~L}$ & joule & $1 \mathrm{~J}=1 \mathrm{~kg}\left(\mathrm{~m} / \mathrm{s}^{2}\right) \cdot \mathrm{m}$ \\
\hline Puissance & $P=W / t$ & $M L^{2} \mathrm{~T}^{-3}$ & watt & $1 \mathrm{~W}=1 \mathrm{~J} / \mathrm{s}$ \\
\hline
\end{tabular}

Il ne faut pas confondre ici les symboles utilisés dans les équations, les symboles représentant les dimensions et les symboles d'unités. Dans l'équation $\mathrm{F}=\mathrm{ma}$, par exemple, «m» représente le terme «masse»; dans la colonne de droite, «m» est le symbole de «mètre», dans le SI

On peut être surpris de voir apparaître le joule et le watt comme unités de mécanique, mais ce joule et ce watt sont exactement les mêmes que l'on retrouve ailleurs, en thermodynamique ou en électricité, par exemple. En fait, le SI est un système d'unités très cohérent. Ainsi, l'énergie électrique consommée, qui est la puissance absorbée pendant un temps donné, pourrait se calculer aussi bien en millions de joules (MJ) qu'en kilowattheures (kW.h) et la puissance d'un moteur automobile pourrait s'exprimer en watts, si nous n'avions pas conservé l'habitude de comparer le nombre de nos «chevaux»!

Le système de mesures anglaises est loin d'offrir la même cohérence et n'est pas aussi unifié. Voici toutefois quelques facteurs de conversion du système anglais au SI, pour les grandeurs déjà mentionnées.

Tableau 2

Unités du système anglais

$\begin{array}{lll}\text { Grandeur } & \text { Unité du système anglais } & \text { Unité équivalente du } \\ \text { Force } & \text { pound ou pound force } & 1 \mathrm{lbf}=4,448 \mathrm{~N} \\ \text { Travail } & \text { foot-pound } & 1 \mathrm{ft}-\mathrm{lb}=1,356 \mathrm{~J} \\ \text { Puissance } & \begin{array}{l}1 \mathrm{ft}-\mathrm{lb} / \mathrm{s}=1,356 \mathrm{~W} \\ \text { foot-pound par seconde } \\ \text { horse power (unité }\end{array} & 1 \mathrm{HP}=550 \mathrm{ft}-\mathrm{lb} / \mathrm{s} \\ & \text { pratique) } & =745 \mathrm{~W} \\ & & =1,014 \mathrm{ch}(*)\end{array}$

(*) $\mathrm{ch}=$ cheval-vapeur ou cheval métrique $(1 \mathrm{ch}=735 \mathrm{~W})$, unité non conforme au SI mais tolérée. Il existe aussi un chevalvapeur, abrégé $\mathrm{CV}$, unité fiscale française sans rapport avec le cheval métrique et qui équivaut à un sixième de litre de cylindrée. CV est parfois utilisé, à tort, comme abréviation de cheval métrique. 


\subsection{MACHINES SIMPLES}

Les machines simples (simple machine, mechanical power) sont des systèmes mécaniques ne comportant qu'un très petit nombre d'organes qui permettent, en exerçant une force motrice (force, motive force, effort) relativement faible, de vaincre une résistance, ou force résistante (resistance, resistive force, load), considérablement plus grande. Le palan, le treuil, le coin et la vis en sont des exemples. Toutes les machines simples sont toutefois des applications de deux principes fondamentaux: le levier (lever) et le plan incliné (inclined plane, wedge). Ces deux principes permettent d'expliquer le fonctionnement de n'importe quel mécanisme, aussi complexe soit-il; ils ont d'ailleurs été découverts très tôt dans l'histoire de l'humanité et ont depuis donné naissance à de nombreuses applications fort ingénieuses.

\subsubsection{Le levier}

Le levier est une barre rigide, mobile autour d'un point d'appui ou axe de rotation (fulcrum) et sur lequel s'exercent, d'une part, la force résistante et, d'autre part, la force motrice. Les distances du point d'appui au point d'application de chacune des forces sont appelées bras de levier (arm, lever arm). Il existe trois genres de leviers.

Dans le levier du premier genre, ou levier inter-appui (first-class lever), le point d'appui est situé entre la résistance et la force motrice (figure 1). On en trouve des applications dans la bascule (seesaw), le levier, ou barre à mine (crowbar), la balance à fléau (beam balance), les ciseaux (scissors), la poulie (pulley, sheave, block), la roue d'engrenage (gear), le treuil (winch, windlass), la pédale de bicyclette, etc.

Le point d'appui du levier du deuxième genre, ou levier inter-résistant (secondclass lever) est situé à l'une des extrémités du levier, la résistance étant placée entre le point d'appui et la force motrice (figure 2). Comme applications, mentionnons la brouette (wheelbarrow), le casse-noix (nut-cracker), le massicot (trimmer), le soufflet (bellows) et la touche de machine à écrire. Les leviers des deux premiers genres sont des mécanismes amplificateurs de force (force-amplifying device) : la force transmise par l'organe «terminal» est plus grande que celle qui est exercée par l'organe «moteur».

Le levier du troisième genre, ou levier inter-moteur, ou encore levier interpuissant (third-class lever) a son point d'appui situé à l'une des extrémités du levier, tandis que la force résistante est placée à l'autre extrémité et que la force motrice s'exerce entre le point d'appui et la résitance (figure 3). Les applications les plus courantes sont les pincettes (nippers, tongs), la pédale de rouet ou de métier à tisser, etc. Dans les leviers de ce genre, il faut exercer une force motrice plus grande que la force résistante. On a donc affaire à des mécanismes amplificateurs de mouvement (motion-amplifying device).

Pour tous les genres de leviers, à l'équilibre, le produit de la résistance, $F_{1}$, par le bras de levier de la résistance (resistance $a r m$ ), $\mathrm{L}_{1}$, est égal au produit de la force motrice, $F_{2}$, par le bras de levier de la force motrice (effort arm), $L_{2}$ :

$$
\mathrm{F}_{1} \cdot \mathrm{L}_{1}=\mathrm{F}_{2} \cdot \mathrm{L}_{2}
$$

Le rapport des forces, ou rapport de multiplication (mechanical advantage), est donc en relation inverse des bras de levier:

$$
\frac{F_{1}}{F_{2}}=\frac{L_{2}}{L_{1}}
$$


Comme illustration du principe du levier, nous examinerons les cas de la poulie, du palan et du treuil. La poulie, tout comme la roue d'engrenage, est une application du levier. En effet, chacun des rayons est un bras de levier de longueur R. Les bras de levier étant égaux, le rapport des forces est égal à $1:$ les forces s'exerçant sur chacun des brins ou garants $(f a l l)$ de la corde sont donc égales. Dans le cas de la poulie fixe (fixed pulley, standing block, single whip), la force résistante s'exerçant sur le brin appelé dormant (standing end) doit être équilibrée par une force motrice d'égale grandeur s'exerçant sur le brin appelé courant (fall, tackle-fall) (figure 4). Notons que la chape (block, shell) de la poulie reçoit, à son point d'ancrage $\mathrm{A}$, la somme des forces résistante et motrice.

Les choses sont différentes dans le cas de la poulie mobile (movable pulley, runner) : la résistance $F_{1}$ se répartit également sur les deux brins, de sorte que le rapport des forces vaut 2 (figure 5). Le dormant supporte en effet la moitié de la résistance $F_{1}$, tandis que le courant supporte l'autre moitié. En contrepartie, toutefois, il faudra tirer 2 mètres de corde pour soulever la résistance de 1 mètre.

Voyons maintenant le cas du palan (pulley-block, block and tackle, hoisting tackle, purchase). Il est constitué d'une moufle fixe (fixed block) et d'une moufle mobile (traveling block), l'une et l'autre constituées d'un certain nombre de poulies réunies dans une même chape. La corde (ou la chaîne) chausse (to reeve) alternativement une poulie de chaque chape. Le palan de la figure 6 comporte trois poulies fixes et trois poulies mobiles. La résistance $F_{1}$ se répartit donc entre les six brins chaussant les poulies de la moufle mobile, de sorte que la force motrice $F_{2}$ qu'il faut exercer sur le courant n'est que de $F_{1} / 6$. Plus généralement, la force motrice qu'il faut exercer sur le courant d'un palan est:

$$
\mathrm{F}_{2}=\mathrm{F}_{1} / 2 \mathrm{n},
$$

n étant le nombre de poulies de la chape mobile. Le principe de la conservation du travail exige toutefois que l'on tire 6 mètres de corde pour soulever la résistance de 1 mètre.

Le treuil (winch, windlass) est constitué de deux poulies de rayons différents sur lesquelles s'exercent les forces résistante et motrice (figure 7). Le rapport des forces résistante et motrice est en proportion inverse des rayons des deux poulies:

$$
\mathrm{F}_{1} / \mathrm{F}_{2}=\mathrm{R}_{2} / \mathrm{R}_{1}
$$

Un engrenage constitué de deux roues de diamètres différents se comporte théoriquement de la même façon que le treuil décrit ici. Tous nos exemples illustrent des leviers du premier genre: ce sont les plus courants en mécanique. Quant aux applications de leviers des deux autres genres, elles obéissent évidemment aux mêmes lois de proportionnalité entre les forces et les déplacements.

\subsubsection{Le plan incliné}

Considérons un corps posé sur un plan incliné (inclined plane, wedge), constitué d'une rampe faisant un angle par rapport à l'horizontale (figure 8 ). Le poids vertical $\mathrm{P}$ de ce corps se décompose en une force $\mathrm{R}$ normale au plan incliné, équilibrée par une réaction d'égale grandeur du plan, mais de sens contraire, et une force $F_{1}$, parallèle au plan incliné et qui déterminera la chute du corps si elle n'est pas compensée par une force motrice au moins équivalente. Chacune des deux forces $R$ et $F_{1}$ est plus petite que $P$. Pour entraîner la résistance vers le haut du plan, il suffit d'opposer à $F_{1}$ une force légèrement plus grande pour rompre l'équilibre (si l'on ne tient pas compte, bien entendu, des frotte- 
ments entre le corps considéré et le plan incliné). Le plan incliné permet donc d'élever une charge en en faisant porter une fraction par le plan lui-même.

La force motrice qu'il faut exercer pour rompre l'équilibre est d'autant plus petite que la pente (slope) est faible. En effet,

$$
\mathrm{F}_{2}=\mathrm{P} \times \text { pente }=\mathrm{P} \times \mathrm{AC} / \mathrm{BC}=\mathrm{P} \times \operatorname{tg} \alpha,
$$

dans laquelle tg $\alpha$ représente la tangente de l'angle que fait le plan par rapport à l'horizontale. Parmi les applications de ce principe, mentionnons le coin (wedge), la came (cam) et la vis (screw).

Le filet d'une vis peut en effet être vu comme un plan incliné enroulé sur un cylindre (figure 9). Une démonstration simple peut nous en convaincre. Si l'on découpe dans une feuille de papier un triangle rectangle que l'on enroule ensuite sur une petite tige cylindrique, on peut constater que l'hypoténuse du triangle décrit sur la tige une hélice (helix). La distance axiale entre chaque spire (turn) est appelée pas (lead, pitch). Chaque spire constitue en effet un petit triangle rectangle ayant pour hauteur le pas, pour base la circonférence de la tige et pour hypoténuse la longueur de la spire.

\subsection{MACHINES COMPOSÉES}

Les machines composées (complex machine) sont des assemblages plus ou moins complexes de machines simples dont l'enchaînement concourt à une transformation ou une transmission de force ou de mouvement. Par exemple, un moteur d'automobile se compose de différents ensembles d'organes (par exemple, l'équipage mobile, le système de distribution, le système d'échappement, etc.). On distingue les machines de transformation d'énergie (prime mover), telles les turbines (turbine), les éoliennes (windmill) ou les moteurs à explosion (internal combustion engine), qui servent à transformer une forme quelconque d'énergie en une autre forme d'énergie, et les machines de production de travail, ou outils (operator), telles les machines agricoles, les appareils électroménagers, etc., qui transforment une forme d'énergie en travail mécanique (figure 10).

Les machines de transformation d'énergie sont celles qui reçoivent une forme quelconque d'énergie naturelle (vent, charbon, énergie hydraulique, uranium fissile, etc.) et la transforment en une énergie mécanique, le plus souvent en un mouvement circulaire (l'organe de sortie est alors un arbre), qui sera utilisée dans des machines de production de travail. Par ailleurs, il peut être commode d'intercaler, entre la machine de transformation d'énergie et la machine de production de travail, d'autres machines réalisant la transmission à distance de formes particulières d'énergie (électrique, hydraulique ou pneumatique, par exemple). Ainsi, la turbine actionne une génératrice qui produit de l"électricité utilisable à des milliers de kilomètres de là par des moteurs transformant l'énergie électrique en énergie mécanique, c'est-à-dire en un mouvement circulaire continu qui servira ultimement à entraîner un outil (figure 11).

\section{TRANSMISSION ET TRANSFORMATION DES FORCES ET DES MOUVEMENTS}

Si l'on se reporte à notre deuxième définition, toute machine comporte un organe d'entrée, ou organe récepteur (intake), un ou plusieurs mécanismes de transformation et de transmission des forces et des mouvements (force and motion modifying and transmitting device) et un organe de sortie, ou organe opérateur (take-off). L'organe récepteur peut être, par exemple, le piston d'une machine à vapeur ou la turbine d'un groupe turbo-générateur de centrale hydraulique; ces deux organes reçoivent une énergie: dans le premier cas l'énergie de pression de la vapeur d'eau, dans le second l'énergie cinétique de l'eau. Dans d'autres machines, l'organe d'entrée est accouplé à l'organe de 
sortie d'une autre machine et reçoit une énergie mécanique : c'est le cas par exemple de la boîte de vitesses d'une automobile, qui est en liaison avec le vilebrequin du moteur. Comme exemple de mécanisme de transformation de mouvement, mentionnons le système bielle-manivelle (sliding-block linkage) du moteur à combustion interne, qui transforme le mouvement rectiligne alternatif (reciprocating motion) des pistons en un mouvement circulaire continu (continuous rotary motion) communiqué à l'arbre de transmission (propeller shaft) par la boîte de vitesses (gear box, transmission) du véhicule. D'autres dispositifs transforment des mouvements circulaires en mouvements rectilignes alternatifs. Pour prendre un autre exemple dans le domaine de la mécanique automobile, mentionnons l'arbre à cames (cam shaft), qui participe au mouvement d'ouverture et de fermeture des soupapes (valve). La boîte de vitesses est, quant à elle, un bon exemple de mécanisme de transformation des forces: en première vitesse, le rapport de démultiplication élevé permet de transmettre aux roues motrices une force considérablement plus grande que celle du moteur. Enfin, comme exemple d'organe de sortie, mentionnons l'arbre moteur (drive shaft), situé dans le prolongement du vilebrequin (crankshaft) du moteur automobile.

L'ensemble des organes concourant à la transmission ou à la transformation d'une force ou d'un mouvement élémentaire constitue un mécanisme, ou système mécanique (mechanism, mechanical system). Les systèmes bielle-manivelle, les trains d'engrenage (gear drive) et les transmissions à chaînes (chain drive) en sont des exemples. La transmission de l'énergie entre la source d'énergie et l'outil peut se faire par une chaîne d'organes mécaniques, auquel cas on parlera de transmission mécanique (mechanical power transmission); elle peut aussi se faire par un fluide, dans les transmissions hydrauliques (hydraulic power transmission) ou pneumatiques (pneumatic power transmission), ou encore par un courant électrique, dans les transmissions électromécaniques (electromechanical power transmission).

\section{OBSERVATIONS TERMINOLOGIQUES}

La terminologie française établit une distinction entre une pièce et un organe. Un organe joue un rôle élémentaire dans la transformation ou la transmission d'une force ou d'un mouvement, tandis qu'une pièce peut être un élément actif (organe) ou passif d'un système mécanique. Par exemple, la roue dentée d'une bicyclette est un organe, tandis que la selle est une pièce. L'anglais ne semble pas faire cette distinction: les termes part, component ou member seront employés indifféremment dans les sens de pièce ou d'organe, sans égard à la fonction.

Divers autres termes d'usage courant ont une signification plus ou moins précise en mécanique. C'est le cas, par exemple, du terme dispositif (device), qui désigne un mécanisme pouvant exécuter, de façon plus ou moins autonome, une opération donnée. Un appareil (device, apparatus, instrument, appliance) est un système mécanique (machine, instrument ou outil) relativement complet en lui-même, participant à l'exécution d'une opération, à la réalisation d'une expérience ou à la fabrication d'un produit.

La chaîne cinématique (kinematic chain) d'une machine est constituée de l'ensemble des organes participant à la transmission et à la transformation d'une force ou d'un mouvement, c'est-à-dire des organes mobiles mis en liaison et limités dans leurs mouvements par des organes fixes : paliers (bearing), coulisseaux (slide), etc., portés par un bâti (frame, chassis). Dans le cas des transmissions mécaniques, les mouvements rectilignes, circulaires ou autres des organes de transmission sont limités ou contenus (constrained) par des organes de guidage appropriés, portés par le bâti de la machine. Ainsi, la chaîne cinématique principale d'un moteur automobile se compose des pistons animés d'un mouvement de va-et-vient (reciprocating motion, to and fro motion, up- 
and-down motion) dans les cylindres du moteur, des bielles (connecting rod) qui transforment ce mouvement alternatif en mouvement circulaire, et du vilebrequin (crankshaft), limité dans ses mouvements par des paliers; celle d'une automobile se compose du moteur, de l'embrayage, de la boîte de vitesses, de l'arbre de transmission, du pont arrière et des roues (figure 12). Chacun de ces sous-ensembles joue un rôle particulier dans la transformation et la transmission des forces et des mouvements.

GILLES BÉLANGER

Université de Montréal, Montréal, Canada

\section{BIBLIOGRAPHIE}

DAVIS, N.L. (1977): Basic Machines : How They Work, Toronto, Coles.

DELANETTE, M. et H. DUBOIS (1969) : Mécanique théorique et appliquée, Aide-mémoire Technor, Paris, Delagrave.

DESTOUCHES, J.-L. (1967) : la Mécanique élémentaire, Paris, PUF.

Dictionnaire encyclopédique Quillet, Quillet, Paris, 1975.

Encyclopédie pratique de mécanique et d'électricité, Quillet, 1965.

Encyclopaedia Universalis, Paris, 1981.

O'BRIEN, R. (1968) : Machines, Time-Life Books.

PEATFIELD, A.E. (1971) : Mechanical Engineering, London, English University Press.

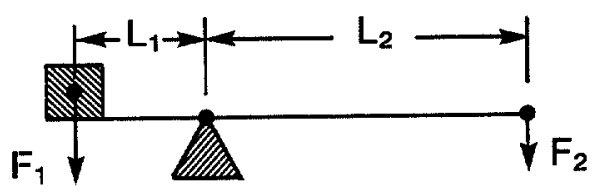

Fig. 1

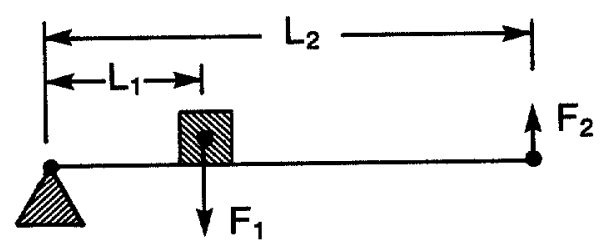

Fig. 2

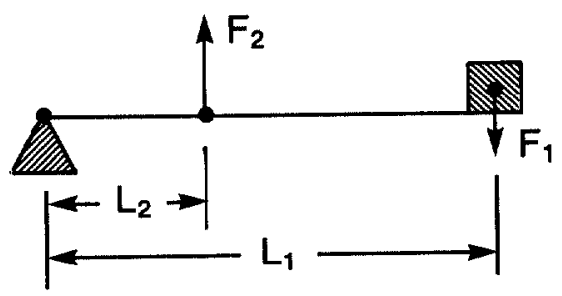

Fig. 3

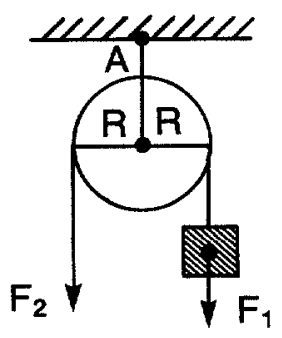

Fig. 4 


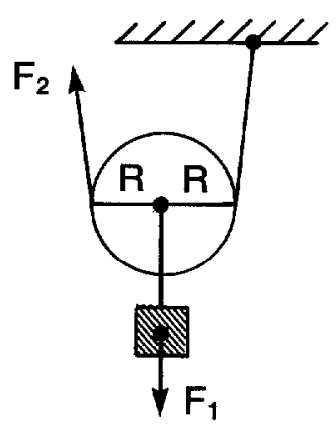

Fig. 5

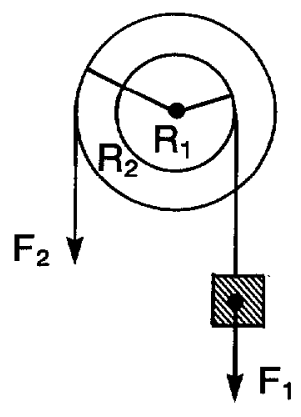

Fig. 7

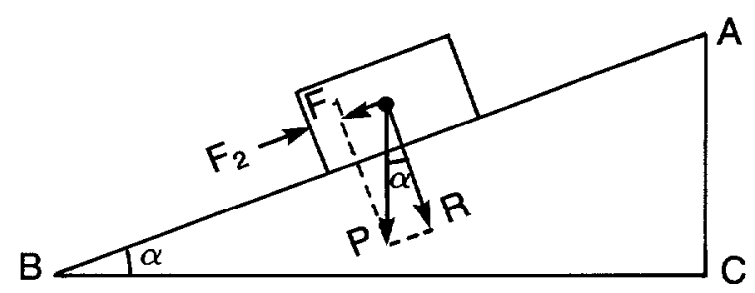

Fig. 8

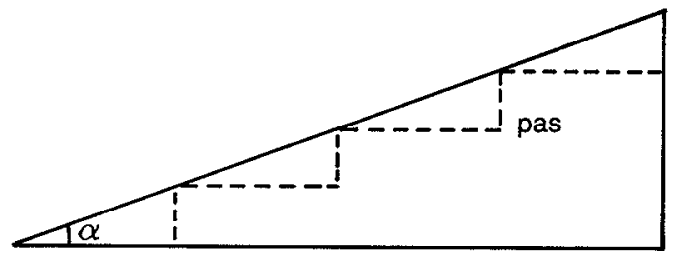

Fig. 9

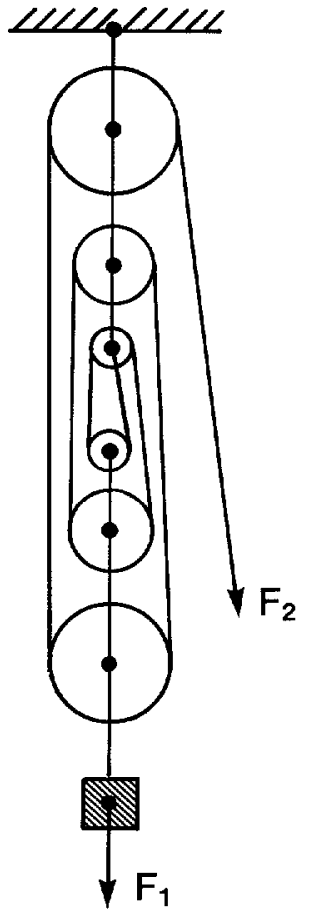

Fig. 6 

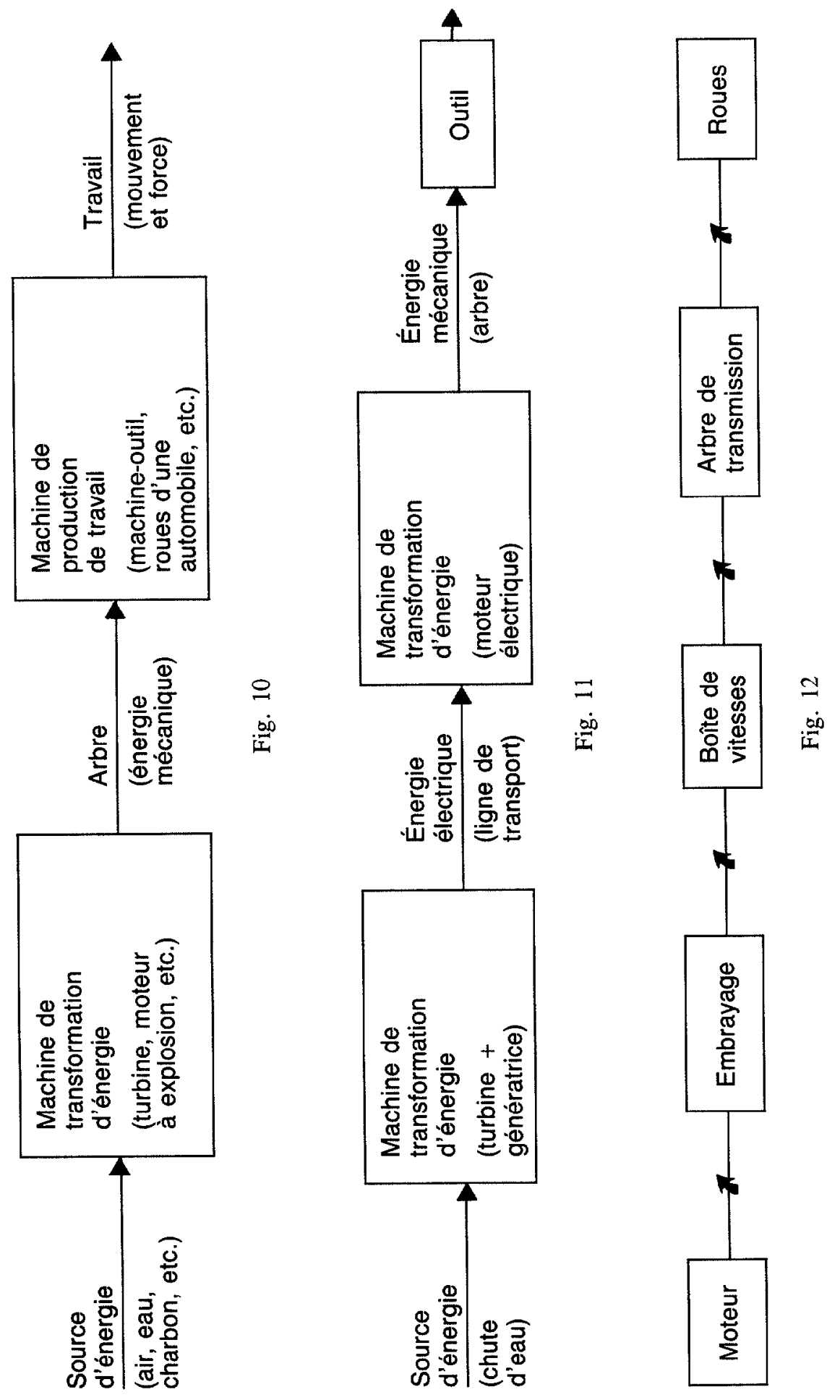